\title{
Initiation and regulation of T-cell responses in tuberculosis
}

\author{
KB Urdahl ${ }^{1,2}$, S Shafiani ${ }^{1}$ and JD Ernst ${ }^{3}$
}

Tuberculosis (TB) poses a great challenge to immunologists, as it represents a chronic infection characterized by persistence of the pathogen despite development of antigen-specific immune responses. Among the characteristics of adaptive immune responses to Mycobacterium tuberculosis is a delay in the onset of detectable T-cell responses, in both humans and experimental animals. Recent studies have revealed mechanisms that contribute to this delay, including pathogen inhibition of apoptosis, delayed migration of dendritic cells from the lungs to the local lymph node, and influence of regulatory T cells. In addition, novel features of $M$. tuberculosis antigen-specific T-cell differentiation have been discovered, which reveal pathways that limit and promote immune control of infection. Taken together, these results highlight the need for additional basic research and provide optimism for the development of TB vaccines with greater efficacy.

\section{INTRODUCTION}

A great challenge in immunology is to understand the complexities, mechanisms, and consequences of host interactions with microbial pathogens. Although all clinical infections are the result of a pathogen's ability to circumvent, overcome, or even exploit innate immune defenses, chronic infections pose additional challenges because of their ability to evade or exploit adaptive immune responses. Mycobacterium tuberculosis, which infects an estimated one of three individuals of the living human population, is an outstanding example of a pathogen that successfully evades adaptive immune responses. Not only does this result in enormous human morbidity and mortality but it also poses unique challenges to the development of an efficacious vaccine to prevent infection or disease.

The past two decades have yielded considerable insights into the pathogenesis of tuberculosis (TB), the mechanisms of protective immunity, and the many ways by which $M$. tuberculosis induces innate and adaptive immune responses and evades elimination by them. In addition, recent studies have provided substantial knowledge of regulation of adaptive immunity in $\mathrm{TB}$, including contexts and mechanisms in which immune responses contribute to tissue injury, morbidity, and even transmission of bacteria to new hosts.

In this review, we have concentrated on recent studies that provide insights into the early cellular and molecular processes underlying the initiation of adaptive immune responses in TB. In particular, these studies have revealed multiple mechanisms that contribute to the long delay between initial infection and onset of detectable cellular immune responses. In addition, we discuss recent work that has provided insights into the mechanisms of regulation of adaptive immunity to $M$. tuberculosis; some of these limit host tissue damage, whereas some provide apparent advantages to the bacteria.

\section{DELAYED INITIAL ACTIVATION OF NAIVE CD4 ${ }^{+}$AND CD8 ${ }^{+} T$ CELLS INTB}

In humans, the onset of adaptive immune responses in TB is considerably delayed compared with responses to other pathogens or inanimate antigens. ${ }^{1}$ Classic studies of TB in isolated communities where there were incidents of brief $(\leqslant 24 \mathrm{~h})$ exposure of naive populations to an active case of pulmonary TB revealed that immune responses to $M$. tuberculosis (measured as skin test reactivity using the Pirquet test or as the appearance of erythema nodosum) occurred an average of 42 days after exposure. ${ }^{2,3}$ Experiments in mice have established that the onset of activation of antigen-specific $T$ cells after aerosol infection with M. tuberculosis is similarly delayed ${ }^{4-9}$ relative to that of other pathogens, such as influenza virus ${ }^{10}$ (Figure 1).

Early insights into the explanation for delayed adaptive immune responses in TB came from the discovery that after

\footnotetext{
${ }^{1}$ Seattle Biomedical Research Institute, Seattle, Washington, USA. ${ }^{2}$ Departments of Immunology and Pediatrics, University of Washington, Seattle, Washington, USA. ${ }^{3}$ Division of Infectious Diseases, Department of Medicine; Departments of Pathology and Microbiology, New York University School of Medicine, New York, New York, USA. Correspondence: KB Urdahl (kevin.urdahl@seattlebiomed.org) or JD Ernst (joel.ernst@med.nyu.edu)

Received 1 January 2011; accepted 31 January 2011; published online 30 March 2011. doi:10.1038/mi.2011.10
} 

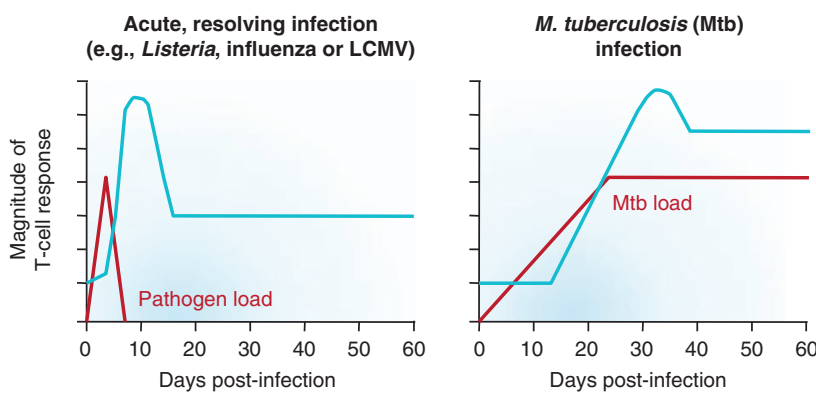

Figure 1 Delayed onset of adaptive immunity in tuberculosis compared with other infections. In acute, resolving infections, T-cell responses are initiated 3-5 days after initial infection; they peak 7-8 days after infection, and subsequently contract to establish memory populations. After aerosol infection with $M$. tuberculosis, T-cell responses are not initiated until 9-11 days after infection, peak several weeks after infection, and bacteria are not eliminated. LCMV, lymphocytic choriomeningitis virus.

aerosol infection, live bacteria must be transported to the lungdraining (also termed "mediastinal," "pulmonary," or "thoracic") lymph node (LDLN) before T-cell priming can occur. Eventually, live bacteria appear in the LDLN between 9 and 11 days after infection, and dissemination to the spleen follows. The activation of $M$. tuberculosis antigen-specific T cells never preceded this dissemination of live bacteria. ${ }^{4}$ Moreover, those same studies revealed that bacteria appeared in the LDLN earlier in a relatively resistant (C57/BL6) strain of mice than in a more susceptible $(\mathrm{C} 3 \mathrm{H} / \mathrm{HeJ})$ strain, indicating the influence of variable host factors on the kinetics of activation of naive $\mathrm{T}$ cells and that earlier activation of naive $T$ cells is associated with superior control of bacterial growth. Subsequent studies that used adoptive transfer of naive $M$. tuberculosis antigen-specific $\mathrm{CD} 4^{+}$ $\mathrm{T}$ cells (from mice with transgenic $\mathrm{T}$-cell antigen receptors specific for ESAT-6 or Ag85B epitopes) demonstrated similar delays in T-cell activation after aerosol infection. ${ }^{5,7,9}$ Furthermore, even fully functional effector Th1 cells transferred into mice and present in the lung parenchyma cannot recognize $M$. tuberculosis antigens and become activated for $>7$ days after aerosol infection, ${ }^{5}$ suggesting that TB bacilli reside in a relatively immuneprivileged site during the earliest stages of infection.

Evidence that suggests that dendritic cell (DC) migration from the lungs to the LDLN is essential for initiation of CD4 ${ }^{+} \mathrm{T}$-cell responses in mice infected with $M$. tuberculosis was first obtained from interleukin (IL)-12p40-deficient mice that revealed a failure of T-cell activation associated with deficient DC migration from the lungs to the LDLN. ${ }^{11}$ The lack of IL-12p40 was associated with the absence of expression of the chemokine receptor, CCR7, on DCs; treatment of DCs with IL-12p40 homodimers restored their ability to migrate to the LDLN and to activate CD4 cells in vivo. The significance of these findings was further strengthened by the use of $M$. tuberculosis that expresses green fluorescent protein, together with flow cytometry, to determine that $\mathrm{CD} 11 \mathrm{c}^{\text {hi }} \mathrm{CD} 11 \mathrm{~b}^{\text {hi }} \mathrm{DCs}$ are a major subset of M. tuberculosisinfected cells in the lungs, and are the nearly exclusive subset of cells that contain bacteria in the LDLN. ${ }^{12}$

Additional studies to understand the mechanisms that account for delayed activation of antigen-specific $\mathrm{CD} 4^{+} \mathrm{T}$ cells in $\mathrm{TB}$ have revealed that a threshold number of bacteria $(\sim 1,500)$ was required in the LDLN to activate naive Ag85B-specific CD4 ${ }^{+}$ $\mathrm{T}$ cells, and that bacteria reach the LDLN from the lungs by transport by $\mathrm{CD} 11 \mathrm{c}^{\text {hi }} \mathrm{CD} 11 \mathrm{~b}^{\text {hi }} \mathrm{DCs},{ }^{9}$ consistent with the observation that IL-12p40-deficient DCs do not migrate to the LDLN or activate $\mathrm{CD} 4^{+} \mathrm{T}$ cells after aerosol infection with $M$. tuberculosis. ${ }^{11}$ These results indicated that DC acquisition of bacteria and/or trafficking may be rate-limiting step(s) in the initiation of adaptive immune responses in TB. Moreover, pulmonary administration of an inflammatory stimulus that recruited DCs to the lungs and drove their migration to the LDLN failed to accelerate the delivery of $M$. tuberculosis to the LDLN or activation of Ag85B-specific CD4 ${ }^{+}$T cells, ${ }^{9}$ indicating that $M$. tuberculosis-infected DCs may be defective in their ability to migrate from the lungs to the LDLN.

\section{M. tuberculosis delays T-cell responses by inhibiting apoptosis}

An important role of $M$. tuberculosis inhibition of apoptosis in delaying and attenuating $\mathrm{CD}^{+}$and $\mathrm{CD} 8^{+} \mathrm{T}$-cell responses has been revealed by several investigations. First, a proapoptotic (SecA2-deficient, resulting in defective secretion of superoxide dismutase A) mutant strain of $M$. tuberculosis promotes the development of antigen-specific $\mathrm{CD} 8^{+} \mathrm{T}$-cell responses and improves control of bacterial growth in vivo. ${ }^{13}$ Second, virulent $M$. tuberculosis manipulates host eicosanoid metabolism to inhibit apoptosis and delay the onset of $\mathrm{CD} 4^{+}$and $\mathrm{CD} 8^{+} \mathrm{T}$-cell responses. Macrophages infected with virulent M. tuberculosis (H37Rv) produce lower amounts of prostaglandin E2 and higher amounts of lipoxin A4 (LXA4) than after infection with an avirulent strain (H37Ra); LXA4 production is associated with an anti-apoptotic, pronecrotic effect. ${ }^{14}$ Moreover, infection of Alox5 (5-lipoxygenase, required for generation of LXA4)-deficient mice with virulent $M$. tuberculosis is associated with a higher frequency of macrophage apoptosis, enhanced DC-dependent cross-priming, accelerated development of antigen-specific $\mathrm{CD} 4^{+}$and $\mathrm{CD} 8^{+}$ $\mathrm{T}$-cell responses, and superior control of the bacterial burden when compared with that of Alox5-sufficient wild-type mice. ${ }^{15}$ The importance of this pathway in regulating immunity to mycobacterial infection was highlighted by a forward genetic screen in zebrafish, which revealed that mutations in lta4h (leukotriene A4 hydrolase) resulting in elevated LXA4 production severely impair host resistance to mycobacterial infection. ${ }^{16}$ Furthermore, findings that polymorphisms in both Alox 5 and lta4h confer susceptibility to human TB confirm the relevance of these pathways to human disease. ${ }^{16,17}$ Thus, inhibition of apoptosis seems to be a major mechanism whereby $M$. tuberculosis delays DC acquisition of bacteria and bacterial antigens and the onset of adaptive immunity. In addition to restricting the initiation of T-cell priming, it seems likely that the ability of M. tuberculosis to inhibit apoptosis may also explain why effector $\mathrm{T}$ cells resident in the lung parenchyma are unable to be activated by $M$. tuberculosis antigens during the first week after aerosol infection, ${ }^{5}$ although this idea has not yet been tested experimentally. Whether additional mechanisms of manipulation of DCs contribute to delays in adaptive immune responses in TB remain to be determined. 


\section{M. tuberculosis-specific Treg cells delay adaptive immunity}

Another likely contributor to delayed T-cell responses during $M$. tuberculosis infection is the early induction of pathogen-specific, Foxp $3^{+}$regulatory T (Treg) cells. Treg cells proliferate and accumulate at sites of infection in both murine $\mathrm{e}^{18,19}$ and human TB. ${ }^{20,21}$ A critical question is whether this Treg cell expansion is simply driven by infection-related inflammation in conjunction with recognition of self-antigens, or whether Treg cells recognize and proliferate in response to $M$. tuberculosis-derived antigens. The initial insight into this question was provided by the observation that expansion of Treg cells, like that of effector T cells, is also delayed. ${ }^{8}$ The inflammation in the lung during the first 2 weeks after aerosol infection with $M$. tuberculosis is not only insufficient to cause Treg cells to undergo enhanced proliferation but proliferation is actually diminished during this time. However, Treg cells undergo robust proliferation, in parallel to effector T cells, after DCs have transported bacilli to the LDLN. ${ }^{8}$ Transfer experiments using $M$. tuberculosis-specific Treg cells

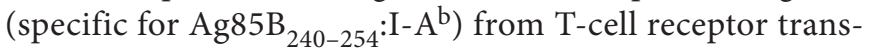
genic mice revealed Treg cell proliferation only in mice infected with wild-type $M$. tuberculosis, but not in mice infected with a virulent strain of $M$. tuberculosis that lacked expression of the specific antigen, Ag85B. ${ }^{8}$ Thus, inflammation associated with M. tuberculosis infection, and IL-2 produced by effector T cells, are both insufficient to drive the non-specific expansion of Treg cells, but Treg cells recognizing $M$. tuberculosis antigens expand preferentially. Moreover, transfer of as few as 40,000 M. tuberculosis-specific Treg cells resulted in an increase in the bacterial burden in the lung, whereas transfer of similar numbers of M. tuberculosis-specific Foxp3-negative CD4 ${ }^{+}$T cells had no effect. ${ }^{8}$ The increased bacterial load in mice receiving $M$. tuberculosisspecific Treg cells was associated with a further delay in the priming of effector T cells in the LDLN and in their subsequent arrival in the lung. This finding is consistent with a previous report in which administration of anti-CD25, a treatment that functionally depletes Treg cells, resulted in increased numbers of M. tuberculosis-specific effector T cells in the lung. ${ }^{22}$ Taken together, these results strongly suggest that Treg cells recognize M. tuberculosis antigens presented by DCs in the LDLN, proliferate, and limit the rate of effector T-cell priming and expansion at this site. The potent ability of small numbers of $M$. tuberculosis Treg cells to restrict effector T-cell priming/expansion may be explained by the previously described ability of activated Treg cells to inhibit the antigen-presenting cell function of DCs. ${ }^{23,24}$ Whether this mechanism is operative during TB needs to be explored.

Although these experiments using Treg cells from T-cell receptor transgenic mice support the existence of $M$. tuberculosisspecific Treg cells, epitopes recognized by endogenous Tregs still need to be defined, not only in TB but also in other infections. ${ }^{25}$ Although support for the existence of pathogen-specific Tregs has been reported in several infections (most clearly in the case of persistent Leishmania major infection $)^{25,26}$ major histocompatibility complex class II tetramers containing epitopes recognized by effector $\mathrm{T}$ cells have failed to identify Foxp $3^{+}$Treg cells in several murine infections, including Listeria mono- cytogenes ${ }^{27}$ and retroviral infections, ${ }^{28}$ suggesting that not every infection may induce pathogen-specific Treg cells. Very little is known about the specificity of Treg cells in any experimental system, and whether M. tuberculosis epitopes recognized by Treg cells are the same, different, or partially overlapping with those recognized by effector $\mathrm{T}$ cells needs to be determined. The immunological pathways manipulated by M. tuberculosis to achieve this induction and to restrict protective immunity should be a rich area for future investigation. Recently, mesenchymal stem cells have been shown to be recruited to sites of M. tuberculosis infection and to suppress T-cell responses, at least in vitro. ${ }^{29}$ In addition, infusion of mesenchymal stem cells into M. tuberculosis-infected mice confers susceptibility to disease that is associated with increased induction of Foxp $3^{+} \mathrm{CD} 4^{+}$ T cells. ${ }^{29}$ Whether mesenchymal stem cells contribute to the induction of $M$. tuberculosis-specific Treg cells requires further investigation.

Are the abilities of $M$. tuberculosis to inhibit apoptosis of infected cells and to induce M. tuberculosis-specific Treg cells two distinct mechanisms by which effector T-cell responses are delayed? Or are they causally related? There are at least two reasons to hypothesize that inhibition of apoptosis, especially by LXA4, may promote expansion of Treg cells. First, inhibition of apoptosis may restrict antigen presentation to infected macrophages and DCs, whereas apoptosis might otherwise promote cross-presentation of $M$. tuberculosis antigens on bystander DCs, which may be uninfected and have more potent stimulatory capacity. Although studies to date have focused on how apoptosis and antigen cross-presentation leads to increased numbers of primed effector $\mathrm{T}$ cells, it seems likely that the types of $\mathrm{T}$ cells induced may also be affected. For example, restricting antigen presentation to infected cells may allow $M$. tuberculosis to better manipulate the type of signals delivered to antigen-specific T cells, which may, in turn, lead to the pathogen-driven expansion of antigen-specific Treg cells. Second, LXA4 induced by virulent strains of $M$. tuberculosis may directly promote the expansion of Treg cells through interactions with the aryl hydrocarbon receptor, $\mathrm{AhR}$, which is a ligand-activated transcription factor expressed in both DCs and T cells. It has a promiscuous ligandbinding site that has been shown to bind multiple synthetic and naturally occurring ligands. ${ }^{30}$ Activation of T cells in the presence of exogenous AhR ligands has been shown to promote the induction of Foxp $3^{+}$Treg cells or Th17 cells, depending on the circumstances, but little is known about the functional importance of natural AhR ligands in T-cell differentiation in vivo. ${ }^{30}$ It is attractive to speculate that $M$. tuberculosis-induced LXA4 may promote the expansion of Treg cells through interactions with AhR in DCs and/or CD4 ${ }^{+}$T cells.

\section{REGULATION OFT-CELL RESPONSES DURING CHRONIC M.TUBERCULOSISINFECTION}

To date, the activity of Treg cells during M. tuberculosis infection has been investigated only during the early immune response, and the data point to their role in delaying T-cell priming in the LDLN. Foxp $3^{+}$Treg cells are also present in the lung, including within granulomas, ${ }^{19}$ and their activity in regulating immune 
responses during chronic infection needs to be explored. However, a recent study has provided insights into other regulatory networks that are operative. Evidence for regulation of T-cell effector phenotypes in the lungs was obtained by studying bone marrow chimeric mice that expressed interferon (IFN) $-\gamma$ receptors (IFN $\gamma$ Rs) on hematopoietic (namely macrophages, DCs, lymphocytes, and neutrophils) but not on non-hematopoietic (namely epithelial, endothelial, smooth muscle, and fibroblast) cells. After infection with M. tuberculosis, these mice were observed to have moderately higher lung bacterial burdens and shortened survival compared with control mice that expressed IFN $\gamma$ Rs on all cells. ${ }^{31}$ In addition, the lungs of M. tuberculosisinfected IFN $\gamma \mathrm{R}$ chimeric mice contained a large excess of neutrophils, indicating markedly dysregulated inflammation. Further investigation revealed marked overexpression of IL-17 in the lungs of IFN $\gamma \mathrm{R}$ chimeric mice, and this was accompanied by overexpression of IL-23, which promotes terminal differentiation and survival of Th17 cells. ${ }^{32} \mathrm{~A}$ role for IFN $\gamma$ signaling was revealed by the findings that IFN $\gamma \mathrm{R}$ chimeric mice markedly underexpressed IFN $\gamma$-responsive gene coding for indoleamine-2,3-dioxygenase, and that specific products of indoleamine-2,3-dioxygenase catabolism of tryptophan inhibited IL-17 expression in vitro, at least in part by blocking the action of IL-23. These results indicate that, in addition to the determinants of T-cell differentiation during initial activation of naive cells in secondary lymphoid organs, the environment of the lungs can also regulate the fate and function of effector cells that are recruited in response to $M$. tuberculosis infection.

\section{MAINTENANCE OFT-CELL RESPONSESTO CHRONIC M.TUBERCULOSISINFECTION}

As in other contexts, such as immunization with foreign proteins, differentiation of naive $\mathrm{T}$ cells into polarized effector cells occurs largely, if not exclusively, in secondary lymphoid tissues in mice infected with $M$. tuberculosis. ${ }^{5}$ However, several observations indicate that maintenance and/or survival of polarized effector $\mathrm{CD} 4^{+}$cells may also occur in the lungs during infection with $M$. tuberculosis.

First, although IL-12 is clearly essential for differentiation of naive $\mathrm{CD} 4^{+} \mathrm{T}$ cells into IFN $\gamma$-producing effector cells in $\mathrm{TB},{ }^{6,33,34}$ strong evidence indicates that continuous expression of IL-12 is required to maintain control of infection. Parenteral administration of IL-12p70 to M. tuberculosis-infected IL12p40-deficient mice restores $\mathrm{CD} 4^{+} \mathrm{T}$-cell production of IFN $\gamma$ and control of bacterial growth in the lungs and spleen, whereas these effects are lost when administration of IL-12p70 is discontinued. ${ }^{35}$ After cessation of IL-12p70 administration, the total number of $\mathrm{CD} 4^{+} \mathrm{T}$ cells in the lungs decreased as did the frequency of IFN $\gamma$-producing $\mathrm{CD} 4^{+} \mathrm{T}$ cells. Furthermore, when $\mathrm{CD} 4^{+} \mathrm{T}$ cells from chronically infected mice were adoptively transferred into M. tuberculosis-infected $R A G 2^{-1-}$ mice, expansion and survival of total $\mathrm{CD} 4^{+} \mathrm{T}$ cells and $\mathrm{CD} 4^{+} \mathrm{T}$ cells capable of producing IFN $\gamma$ were much poorer in IL-12p40-deficient $R A G 2^{-/-}$recipients than in IL-12-sufficient $R A G 2^{-/-}$recipients. These studies clearly indicate a role for IL- 12 in the maintenance of differentiated Th1 effector cells, and likely also in the overall survival of Th1 cells. Whether the chronic effects of IL12 on lung $\mathrm{CD}^{+} \mathrm{T}$ cells are due solely to IL- 12 expression in the lungs was not determined, although IL-12 is clearly expressed in the lungs of $M$. tuberculosis-infected mice. ${ }^{36}$

Although T-cell responses need to be maintained for M. tuberculosis to be controlled during chronic disease, the degree to which $M$. tuberculosis antigens recognized by $\mathrm{T}$ cells change between acute and chronic stages of TB is currently unclear. Some antigenic proteins, such as Ag85B, are primarily expressed during the early stages of $M$. tuberculosis infection, ${ }^{37}$ and $\mathrm{T}$ cells recognizing Ag85B, and other antigens expressed with similar kinetics, may not contribute to protection during chronic infection. Other antigenic proteins (such as ESAT-6), seem to be recognized by antigen-specific $\mathrm{T}$ cells during both acute and chronic stages of disease. ${ }^{38} \mathrm{~T}$ cells recognizing ESAT6 express cell surface markers with characteristics of effector $\mathrm{T}$ cells and undergo high levels of proliferation, both suggesting chronic antigenic stimulation. ${ }^{39} \mathrm{~A}$ third group of $\mathrm{T}$ cells may recognize $M$. tuberculosis antigens expressed during chronic but not acute infection, but this group of T cells is the least well characterized. ${ }^{40,41}$ The relative contribution of each of these types of $\mathrm{T}$ cells to protective immunity during each stage of infection remains poorly understood, but has obvious implications for vaccine design.

Typically, effector $\mathrm{T}$ cells are short-lived cells that die after a period of robust antigen-driven proliferation. ${ }^{42}$ Long-lived antigen-specific memory $\mathrm{T}$ cells, by definition, are only generated after antigen has been cleared. Memory $T$ cells are maintained by a slow, but a steady rate of proliferation that is not antigen dependent. ${ }^{42}$ How then, are high numbers of rapidly dividing, antigenspecific $\mathrm{T}$ cells maintained during persistent $M$. tuberculosis infection? Why do M. tuberculosis-specific T cells not succumb to the same fate as antigen-specific $\mathrm{T}$ cells in a well-studied chronic viral infection (i.e., chronic lymphocytic choriomeningitis virus infection), in which antigenic T cells gradually lose effector T-cell function, become anergic, and then die? ${ }^{43,44}$ Importantly, maintaining antigen-specific T cells during M. tuberculosis infection does not require the generation of new $\mathrm{T}$ cells from the thymus, as even thymectomized animals maintain near-normal T-cell responses during persistent infection. ${ }^{38}$ This is fortuitous, as M. tuberculosis has been located in DCs in the thymic medulla, raising the possibility that newly developing $M$. tuberculosisspecific T cells may be deleted during thymic development. ${ }^{45}$

A recent study has begun to address these questions by identifying subsets of antigen-specific $\mathrm{CD} 4^{+} \mathrm{T}$ cells with distinct functional properties. ${ }^{39}$ Most antigen-specific (i.e., ESAT- $6_{4-17^{-}}$ specific) $\mathrm{CD} 4^{+} \mathrm{T}$ cells, defined by a major histocompatibility complex class II tetramer, were found to express cell surface markers previously associated with effector T-cell functional exhaustion (programmed death-1 (PD-1)), or terminal differentiation (killer cell lectin-like receptor G1 (KLRG1)). Although a few antigen-specific $\mathrm{T}$ cells expressed both of these receptors, most were either PD- $1^{+} \mathrm{KLRG} 1$ or PD- $1^{-} \mathrm{KLRG}^{+}$, and these sub-populations had unique functional properties. $\mathrm{PD}-1^{+}$ T cells exhibited a high degree of proliferation, whereas KLRG1 ${ }^{+}$ T cells proliferated less, but expressed high levels of IFN- $\gamma$ and 
tumor necrosis factor- $\alpha .{ }^{39}$ Adoptive transfer studies demonstrated that $\mathrm{PD}-1^{+} \mathrm{T}$ cells were maintained at high levels and a subset differentiated into KLRG1 ${ }^{+} \mathrm{T}$ cells. In contrast, KLRG1 ${ }^{+}$ $\mathrm{T}$ cells maintained their phenotype, but underwent rapid attrition..$^{39}$ These results support a model in which antigen-specific $\mathrm{PD}-1^{+} \mathrm{CD} 4{ }^{+} \mathrm{T}$ cells represent a proliferating precursor population that serves as an "engine" for the T-cell response. They maintain a high degree of proliferative capacity and have the potential to give rise to more terminally differentiated effector $\mathrm{T}$ cells. More understanding of the functional characteristics of these PD $-1^{+} \mathrm{T}$ cells is critical. Cellular and molecular signals that facilitate their survival, as well as signals that prevent, and eventually facilitate, terminal differentiation in a subset of these cells need to be identified.

KLRG1 ${ }^{+} \mathrm{T}$ cells in TB have properties consistent with those demonstrated by KLRG1 ${ }^{+} \mathrm{T}$ cells in other infections and experimental systems; they are terminally differentiated effector $\mathrm{T}$ cells that produce effector cytokines, but have a low proliferative capacity and a short lifespan. ${ }^{46,47}$ In contrast, the properties of PD-1 ${ }^{+} \mathrm{T}$ cells in $\mathrm{TB}$ are surprisingly different from previous studies. In chronic lymphocytic choriomeningitis virus infection, e.g., PD-1 is expressed on functionally exhausted T cells, and blockade of PD-1-programmed cell death ligand 1 leads to improved T-cell function and enhanced viral clearance. ${ }^{48}$ However, in TB, PD-1-expressing T cells are not functionally anergic, but undergo robust proliferation and seem to be central to maintaining a productive effector T-cell response. ${ }^{39} \mathrm{~A}$ positive role for PD-1 in promoting immunity during TB is supported by the recent finding that $\mathrm{PD}-1^{-/-}$mice are extremely susceptible to $M$. tuberculosis infection with exacerbated immunopathology and high bacterial burdens in the lung. ${ }^{49,50}$ This immunopathology is associated with a profound expansion of antigen-specific $\mathrm{CD}^{+} \mathrm{T}$ cells and extremely elevated secretion of proinflammatory cytokines, and depletion of $\mathrm{CD} 4^{+} \mathrm{T}$ cells rescues $\mathrm{PD}-1$ deficient mice from early death. ${ }^{50}$ Putting these results together with the finding that $\mathrm{PD}-1^{+} \mathrm{CD} 4^{+} \mathrm{T}$ cells represent a proliferating precursor population, it is attractive to speculate that $\mathrm{PD}-1$ functions to restrain $\mathrm{CD} 4^{+} \mathrm{T}$ cells from fully differentiating, which is critical for preventing deleterious immunopathology, maintaining long-term populations of antigen-specific $\mathrm{T}$ cells, and ultimately for controlling $M$. tuberculosis infection.

\section{CONCLUSIONS: RELEVANCE FOR IMMUNIZATION STRATEGIES}

The immune response to TB is a tightly regulated process, at least some of which is driven by $M$. tuberculosis to facilitate persistent infection. This probably explains why the rate at which adaptive immunity is initiated has been found to be so critical for successful immune protection; ${ }^{51}$ if effector T cells can be induced early, perhaps immune control can be established before $M$. tuberculosis can effectuate an array of pathogen-beneficial regulatory networks. To avert this early control, virulent M. tuberculosis has devised multiple strategies to slow the initial T-cell response. Particularly daunting to immunization efforts is the ability of M. tuberculosis to evade recognition by effector $\mathrm{T}$ cells in the lung parenchyma for at least a week after aerosol infection, ${ }^{5}$ which helps to explain why parenterally administered vaccines have no effect on the replication of $M$. tuberculosis in the lungs for 2 weeks after infection. ${ }^{52}$ Recent studies showing that mucosally administered vaccines induce airway luminal effector T cells that are capable of curbing $M$. tuberculosis replication much earlier offer promise as an adjunct to parental vaccines. ${ }^{52-54}$ The finding that $M$. tuberculosis-specific $\mathrm{CD} 4^{+}$ $\mathrm{T}$ cells are maintained through a $\mathrm{PD}-1^{+}$proliferative precursor population ${ }^{39}$ also seems highly relevant for vaccine design. A vaccine that induces antigen-specific $\mathrm{CD} 4^{+} \mathrm{T}$ cells capable of becoming PD- $1^{+}$cells with the potential for self-renewal may lead to enhanced long-term protection compared with a vaccine that simply generates large numbers of short-lived terminally differentiated effector T cells. Many puzzling questions regarding T-cell-mediated immunity to TB are starting to be solved, but a more fundamental understanding is required to inform the rational design of an effective vaccine.

\section{ACKNOWLEDGMENTS}

This study was supported in part by grants from the National Institutes of Health: R01 Al076327 (KBU), R01 Al051242, and R01 Al084041 (JDE).

\section{DISCLOSURE}

The authors declared no conflict of interest.

\section{(c) 2011 Society for Mucosal Immunology}

\section{REFERENCES}

1. Miller, J.D. et al. Human effector and memory CD8 ${ }^{+}$T-cell responses to smallpox and yellow fever vaccines. Immunity 28, 710-722 (2008).

2. Poulsen, A. Some clinical features of tuberculosis I. Incubation period. Acta. Tuberculosea Scandinavica 24, 311-346 (1950).

3. Wallgren, A. The time-table of tuberculosis. Tubercle 29, 245-251 (1948).

4. Chackerian, A.A., Alt, J.M., Perera, T.V., Dascher, C.C. \& Behar, S.M. Dissemination of Mycobacterium tuberculosis is influenced by host factors and precedes the initiation of T-cell immunity. Infect. Immun. 70, 4501-4509 (2002).

5. Gallegos, A.M., Pamer, E.G. \& Glickman, M.S. Delayed protection by ESAT-6-specific effector CD4 ${ }^{+}$T cells after airborne $M$. tuberculosis infection. J. Exp. Med. 205, 2359-2368 (2008).

6. Khader, S.A. et al. IL-23 compensates for the absence of IL-12p70 and is essential for the IL-17 response during tuberculosis but is dispensable for protection and antigen-specific IFN-gamma responses if IL-12p70 is available. J. Immunol. 175, 788-795 (2005).

7. Reiley, W.W. et al. ESAT-6-specific CD4+ T-cell responses to aerosol Mycobacterium tuberculosis infection are initiated in the mediastinal lymph nodes. Proc. Natl Acad. Sci. USA 105, 10961-10966 (2008).

8. Shafiani, S., Tucker-Heard, G., Kariyone, A., Takatsu, K. \& Urdahl, K.B. Pathogen-specific regulatory $T$ cells delay the arrival of effector $T$ cells in the lung during early tuberculosis. J. Exp. Med. 207, 1409-1420 (2010).

9. Wolf, A.J. et al. Initiation of the adaptive immune response to Mycobacterium tuberculosis depends on antigen production in the local lymph node, not the lungs. J. Exp. Med. 205, 105-115 (2008).

10. Lawrence, C.W. \& Braciale, T.J. Activation, differentiation, and migration of naive virus-specific CD8 ${ }^{+}$T-cells during pulmonary influenza virus infection. J. Immunol. 173, 1209-1218 (2004).

11. Khader, S.A. et al. Interleukin 12 p40 is required for dendritic cell migration and $T$ cell priming after Mycobacterium tuberculosis infection. J. Exp. Med. 203, 1805-1815 (2006).

12. Wolf, A.J. et al. Mycobacterium tuberculosis infects dendritic cells with high frequency and impairs their function in vivo. J. Immunol. 179, 2509-2519 (2007).

13. Hinchey, J. et al. Enhanced priming of adaptive immunity by a proapoptotic mutant of Mycobacterium tuberculosis. J. Clin. Invest. 117, 2279-2288 (2007). 
14. Chen, $M$. et al. Lipid mediators in innate immunity against tuberculosis: opposing roles of PGE2 and LXA4 in the induction of macrophage death. J. Exp. Med. 205, 2791-2801 (2008).

15. Divangahi, M., Desjardins, D., Nunes-Alves, C., Remold, H.G. \& Behar, S.M. Eicosanoid pathways regulate adaptive immunity to Mycobacterium tuberculosis. Nat. Immunol. 11, 751-758 (2010).

16. Tobin, D.M. et al. The Ita4h locus modulates susceptibility to mycobacterial infection in zebrafish and humans. Cell 140, 717-730 (2010).

17. Herb, F. et al. ALOX5 variants associated with susceptibility to human pulmonary tuberculosis. Hum. Mol. Genet. 17, 1052-1060 (2008).

18. Kursar, M. et al. Cutting edge: regulatory T cells prevent efficient clearance of Mycobacterium tuberculosis. J. Immunol. 178, 2661-2665 (2007).

19. Scott-Browne, J.P. et al. Expansion and function of Foxp3-expressing T regulatory cells during tuberculosis. J. Exp. Med. 204, 2159-2169 (2007).

20. Guyot-Revol, V., Innes, J.A., Hackforth, S., Hinks, T. \& Lalvani, A. Regulatory $T$ cells are expanded in blood and disease sites in patients with tuberculosis. Am. J. Respir. Crit. Care Med. 173, 803-810 (2006).

21. Ribeiro-Rodrigues, $\mathrm{R}$. et al. A role for $\mathrm{CD} 4{ }^{+} \mathrm{CD} 25^{+} \mathrm{T}$ cells in regulation of the immune response during human tuberculosis. Clin. Exp. Immunol. 144, 25-34 (2006)

22. Quinn, K.M. et al. Inactivation of $\mathrm{CD} 4{ }^{+} \mathrm{CD} 25^{+}$regulatory T cells during early mycobacterial infection increases cytokine production but does not affect pathogen load. Immunol. Cell Biol. 84, 467-474 (2006).

23. Onishi, Y., Fehervari, Z., Yamaguchi, T. \& Sakaguchi, S. Foxp3+ natural regulatory $T$ cells preferentially form aggregates on dendritic cells in vitro and actively inhibit their maturation. Proc. Natl Acad. Sci. USA 105, 10113-10118 (2008).

24. Wing, K. et al. CTLA-4 control over Foxp3+ regulatory T-cell function. Science 322, 271-275 (2008).

25. Belkaid, Y. \& Tarbell, K. Regulatory T cells in the control of hostmicroorganism interactions (*). Annu. Rev. Immunol. 27, 551-589 (2009).

26. Suffia, I.J., Reckling, S.K., Piccirillo, C.A., Goldszmid, R.S. \& Belkaid, Y. Infected site-restricted Foxp3+ natural regulatory $T$ cells are specific for microbial antigens. J. Exp. Med. 203, 777-788 (2006)

27. Ertelt, J.M., Rowe, J.H., Johanns, T.M., Lai, J.C., McLachlan, J.B. \& Way, S.S. Selective priming and expansion of antigen-specific Foxp3- CD4 ${ }^{+}$ T-cells during Listeria monocytogenes infection. J. Immunol. 182, 3032-3038 (2009)

28. Antunes, I. et al. Retrovirus-specificity of regulatory T cells is neither present nor required in preventing retrovirus-induced bone marrow immune pathology. Immunity 29, 782-794 (2008).

29. Raghuvanshi, S., Sharma, P., Singh, S., Van Kaer, L. \& Das, G. Mycobacterium tuberculosis evades host immunity by recruiting mesenchymal stem cells. Proc. Natl Acad. Sci. USA 107, 21653-21658 (2010).

30. Ho, P.P. \& Steinman, L. The aryl hydrocarbon receptor: a regulator of Th17 and Treg cell development in disease. Cell Res. 18, 605-608 (2008)

31. Desvignes, L. \& Ernst, J.D. Interferon-gamma-responsive nonhematopoietic cells regulate the immune response to Mycobacterium tuberculosis. Immunity 31, 974-985 (2009).

32. McGeachy, M.J. et al. The interleukin 23 receptor is essential for the terminal differentiation of interleukin 17-producing effector Thelper cells in vivo. Nat. Immunol. 10, 314-324 (2009)

33. Cooper, A.M., Kipnis, A., Turner, J., Magram, J., Ferrante, J. \& Orme, I.M. Mice lacking bioactive IL-12 can generate protective, antigen-specific cellular responses to mycobacterial infection only if the IL-12 p40 subunit is present. J. Immunol. 168, 1322-1327 (2002).

34. Cooper, A.M., Magram, J., Ferrante, J. \& Orme, I.M. Interleukin 12 (IL-12) is crucial to the development of protective immunity in mice intravenously infected with Mycobacterium tuberculosis. J. Exp. Med. 186, 39-45 (1997).
35. Feng, C.G. et al. Maintenance of pulmonary Th1 effector function in chronic tuberculosis requires persistent IL-12 production. J. Immunol. 174, 4185-4192 (2005).

36. Jung, Y.J., LaCourse, R., Ryan, L. \& North, R.J. Evidence inconsistent with a negative influence of $T$ helper 2 cells on protection afforded by a dominant Thelper 1 response against Mycobacterium tuberculosis lung infection in mice. Infect. Immun. 70, 6436-6443 (2002).

37. Rogerson, B.J., Jung, Y.J., LaCourse, R., Ryan, L., Enright, N. \& North, R.J. Expression levels of Mycobacterium tuberculosis antigen-encoding genes versus production levels of antigen-specific T-cells during stationary level lung infection in mice. Immunology 118, 195-201 (2006).

38. Winslow, G.M., Roberts, A.D., Blackman, M.A. \& Woodland, D.L. Persistence and turnover of antigen-specific CD4 ${ }^{+}$T-cells during chronic tuberculosis infection in the mouse. J. Immunol. 170, 2046-2052 (2003).

39. Reiley, W.W. et al. Distinct functions of antigen-specific CD4 T cells during murine Mycobacterium tuberculosis infection. Proc. Natl Acad. Sci. USA 107, 19408-19413 (2010)

40. Black, G.F. et al. Immunogenicity of novel DosR regulon-encoded candidate antigens of Mycobacterium tuberculosis in three high-burden populations in Africa. Clin. Vaccine Immunol. 16, 1203-1212 (2009).

41. Leyten, E.M. et al. Human T-cell responses to 25 novel antigens encoded by genes of the dormancy regulon of Mycobacterium tuberculosis. Microbes Infect. 8, 2052-2060 (2006).

42. Sallusto, F., Lanzavecchia, A., Araki, K. \& Ahmed, R. From vaccines to memory and back. Immunity 33, 451-463 (2010).

43. Wherry, E.J., Blattman, J.N., Murali-Krishna, K., van der Most, R. \& Ahmed, R. Viral persistence alters CD8 T cell immunodominance and tissue distribution and results in distinct stages of functional impairment. J. Virol. 77, 4911-4927 (2003).

44. van der Most, R.G. et al. Changing immunodominance patterns in antivira CD8 T-cell responses after loss of epitope presentation or chronic antigenic stimulation. Virology 315, 93-102 (2003).

45. Nobrega, C. et al. Dissemination of mycobacteria to the thymus renders newly generated T cells tolerant to the invading pathogen. J. Immunol. 184, 351-358 (2010).

46. Voehringer, D., Blaser, C., Brawand, P., Raulet, D.H., Hanke, T. \& Pircher $\mathrm{H}$. Viral infections induce abundant numbers of senescent CD8 T cells. J. Immunol. 167, 4838-4843 (2001)

47. Joshi, N.S. et al. Inflammation directs memory precursor and short-lived effector CD8(+) T-cell fates via the graded expression of T-bet transcription factor. Immunity 27, 281-295 (2007).

48. Barber, D.L. et al. Restoring function in exhausted CD8 T cells during chronic viral infection. Nature 439, 682-687 (2006)

49. Lázár-Molnár, E. et al. Programmed death-1 (PD-1)-deficient mice are extraordinarily sensitive to tuberculosis. Proc. Natl Acad. Sci. USA 107, 13402-13407 (2010)

50. Barber, D.L., Mayer-Barber, K.D., Feng, C.G., Sharpe, A.H. \& Sher, A. CD4 T cells promote rather than control tuberculosis in the absence of PD-1-mediated inhibition. J. Immunol. 186, 1598-1607 (2010).

51. Cooper, A.M. Cell-mediated immune responses in tuberculosis. Annu. Rev. Immunol. 27, 393-422 (2009).

52. Jeyanathan, M., Heriazon, A. \& Xing, Z. Airway luminal T cells: a newcomer on the stage of TB vaccination strategies. Trends Immunol. 31, 247-252 (2010).

53. Jeyanathan, M. et al. Murine airway luminal antituberculosis memory CD8 T cells by mucosal immunization are maintained via antigen-driven in situ proliferation, independent of peripheral T-cell recruitment. Am. J. Respir. Crit. Care Med. 181, 862-872 (2010).

54. Ronan, E.O., Lee, L.N., Beverley, P.C. \& Tchilian, E.Z. Immunization of mice with a recombinant adenovirus vaccine inhibits the early growth of Mycobacterium tuberculosis after infection. PLoS One 4, e8235 (2009). 